\title{
Labyrinthe
}

$10 \mid 2001$

Numéro 10

\section{Entre abbreviatio et auctoritas}

Traduire et commenter la Bible dans un texte médiéval anglais

Jean-Pascal Pouzet

\section{(2) OpenEdition}

Journals

Édition électronique

URL : http://journals.openedition.org/labyrinthe/1203

DOI : $10.4000 /$ labyrinthe. 1203

ISSN : 1950-6031

Éditeur

Hermann

Édition imprimée

Date de publication : 1 octobre 2001

Pagination : 29-38

Référence électronique

Jean-Pascal Pouzet, "Entre abbreviatio et auctoritas », Labyrinthe [En ligne], 10 | 2001, mis en ligne le 08 avril 2006, consulté le 21 avril 2019. URL : http://journals.openedition.org/labyrinthe/1203 ; DOI :

10.4000/labyrinthe. 1203

Ce document a été généré automatiquement le 21 avril 2019.

Propriété intellectuelle 


\title{
Entre abbreviatio et auctoritas
}

Traduire et commenter la Bible dans un texte médiéval anglais

\author{
Jean-Pascal Pouzet
}

Le manuscrit Oxford, Trinity College 93 contient le seul exemplaire connu d'une longue composition en prose moyen anglaise, dont l'examen approfondi s'avère indispensable pour une meilleure connaissance des «mises en anglais » de la Bible dans l'Angleterre médiévale, et singulièrement à la charnière des $\mathrm{XIV}^{\mathrm{e}}$ et $\mathrm{XV}^{\mathrm{e}}$ siècles ${ }^{1}$. Écrit dans un module serré par une main unique, qui couvre 200 folios d'un volume de petit format, ce document a été jusqu'ici fort peu étudié par la critique, bien que son existence, à une époque travaillée par la légitimité de la traduction de la Bible en langue vernaculaire, eût pu susciter plus de questionnements. Littéralement redécouvert en 1960, le manuscrit a été édité par R. Reilly dans une thèse demeurée inédite²

2 Le titre sous lequel il est le plus généralement connu dans la critique, le désigne sous le nom de « résumé de la Bible en moyen anglais » (« Middle English Summary of the Bible »). Ce titre n'est pas adéquat: si le texte est certes un « résumé ", là n'est pas toute sa nature. Car ce mot éclipse l'autre composante de son économie, celle qui repose sur l'insertion d'éléments de commentaire fonctionnant à plusieurs niveaux, dont le principal est sans doute la référence à des autorités citées et traduites - des auctoritates, selon le nom consacré par la recherche sur les sources et les usages du savoir scholastique médiéval. Pour tenter de comprendre ce double fonctionnement, j'adopte le vocabulaire d'analyse suivant: plutôt que de parler exclusivement de "résumé » ou de "commentaire », je cherche à rendre compte de l'interaction entre ces deux composantes, et je désigne le texte transmis par ce manuscrit d'Oxford sous le terme relativement neutre de compendium. Je rassemble tout ce qui a trait au « résumé » sous le vocable d'abbreviatio, et tout ce qui ressortit au « commentaire » sous celui d'auctoritas.

3 Le compendium appartient à la riche production religieuse en langue anglaise de son temps. La place manque pour camper le tableau de cette littérature vernaculaire à la fin du XIV : on évoquera seulement que l'« âge d'or ", dont parle N. Watson, est aussi une véritable "ère du soupçon", celui que les autorités ecclésiastiques font peser sur un corpus qu'elles voient leur échapper, à la suite des prises de position d'un des plus éminents théologiens de sa génération, John Wyclif, et du mouvement de traduction de la 
Bible en langue latine (la «Vulgate » de saint Jérôme) entrepris par ceux que l'on appelle les Lollards ${ }^{3}$. Il est certain que les techniques de traduction et de commentaire sont parmi les éléments d'où part la controverse religieuse anglaise; mon propos est d'évoquer le mode de fonctionnement du texte considéré, en cherchant à définir sa place dans le paysage vernaculaire général.

Techniques d'abbreviatio

4 C'est bien du texte complet de la Bible que part le compilateur, comme en témoignent de nombreux fragments que le copiste du manuscrit (peut-être la même personne que notre auteur) souligne en rouge dans la plupart des cas. La mise en page du texte entend ainsi rendre matériel le processus complexe de lecture-assimilation et de commentaire. Mais le texte sacré n'est présent que sous cette forme de citations brèves : il est littéralement « abrégé ». Dans ses moindres détails, le style auquel a recours le compilateur ressortit à une stratégie de composition dans laquelle chacun des lemmes bibliques est l'objet d'une rigoureuse "mise en format ». Comparons ainsi quelques-uns des vers qui décrivent l'Épouse du Cantique des Cantiques (7. 1-8, 13), avec ce qu'il en advient dans le compendium :

(1) [...] quam pulchri sunt gressus tui in calciamentis filia principis

iunctura feminum tuorum sicut monilia quae fabricata sunt manu artificis

(2) umbilicus tuus crater tornatilis numquam indigens poculis

venter tuus sicut acervus tritici vallatus liliis

(3) duo ubera tua sicut duo hinuli gemelli capreae

(4) collum tuum sicut turris eburnea

oculi tui sicut piscinae in Esebon quae sunt in porta filiae multitudinis

nasus tuus sicut turris Libani quae respicit contra Damascum

(5) caput tuum ut Carmelus et comae capitis tui sicut purpura regis

vincta canalibus

(6) quam pulchra es et quam decora carissima in deliciis

(7) statura tua adsimilata est palmae et ubera tua botris

(8) dixi ascendam in palmam adprehendam fructus eius et erunt ubera tua sicut botri vineae $[. .$.

(13) [...] omnia poma nova et vetera dilecte mi servabi tibi ${ }^{4}$.

Le texte retenu ici est celui de la Bible Vulgate. Si la langue du texte de la Bible qui a servi de « support » au compilateur anglais est un problème essentiel qui occupe la fin de mon propos, les remarques sur la nature de son intervention seraient les mêmes en référence à un texte biblique en langue anglaise. Observons pour le moment que la version moyen anglaise va loin dans la condensation syntaxique et rythmique :

Ful feyre bene thi goings, the ioyntours of thi hippes, thi nauel, thi wombe, pappes, nek, eghne, nees, heed, heres. This stature is likkend to a palm tre. I shal stegh in to

a palme tre etc. Pappes as clusters. [...] Alle appels nwe and olde etc. ${ }^{5}$

Chacun des syntagmes nominaux, construits en apposition et tous sujets du prédicat « pulchri sunt » (figurés en caractères gras dans l'extrait en latin), est réduit à sa plus simple expression, le noyau nominal stricto sensu. L'effet de parataxe des versets est accentué par la nouvelle série appositive (mise en italique dans le passage en anglais), où les comparaisons introduites en latin par «sicut» et «ut» n'ont plus de place - seul le groupe verbal «is likkend», traduisant "adsimilata est», rend un écho de la modalité comparative. Mais au-delà des ressources proprement morphosyntaxiques, on remarquera aussi l'usage de l'abréviation "etc.", à deux reprises (en gras). La consultation du compendium montre bien vite que cet élément, importé de la culture latine, est de loin l'outil abrégeant le plus fréquent: ses occurrences se comptent littéralement par centaines. Il est curieux de noter que « etc. » n'est pas utilisé à la seule 
fin de « couper court » à un long développement. Bien au contraire, comme ici, il sert à rendre compte in absentia d'unités textuelles très petites, si bien que son usage pourrait apparaître disproportionné, par rapport à l'économie réelle en nombre de mots (souvent infime en réalité), si l'on n'y voyait pas surtout un procédé systématique de présentation du texte sacré - sinon d'intervention sur lui, toute déférente qu'elle soit.

7 Mon choix du terme abbreviatio n'est pas innocent. L'abbreviatio correspond, avec son antonyme l'amplificatio, à l'un des deux principaux composants de l'elocutio, ou style, luimême l'un des cinq éléments traditionnels de la rhétorique. Ce terme convoque ainsi non seulement une longue tradition intellectuelle pour l'Occident, mais surtout une délicate question qui traverse cette tradition, celle de la nature et de la place de la rhétorique. Rita Copeland démontre en effet que la rhétorique, par rapport à ses premières définitions chez les auteurs latins antiques (Cicéron, Horace, Quintilien), ne cesse d'agrandir son territoire discursif au cours des siècles, pour tenir au Moyen Âge une place prépondérante dans la recherche et la formulation du sens visé par le commentaire dont l'exégèse de la Bible, qui est le Commentaire par excellence. C'est à cette «montée en puissance » de la rhétorique et de ses enjeux que renvoient les dispositifs du texte: toujours selon Copeland, l'abbreviatio est l'un des moyens par lesquels le poète anglais Geoffrey Chaucer, dans la Légende des femmes vertueuses, poème contemporain du compendium, marque ses distances avec ses sources, ses propres auctoritates, pour faire de la rhétorique un principe de liberté créatrice, et devenir l'auctor de son texte 6 . Si le rapprochement tenté ici avec Chaucer, le "grans translateur» célébré par Eustache Deschamps dans une ballade célèbre, pose autant de problèmes qu'il peut en éclairer, l' abbreviatio à laquelle a recours le compilateur du manuscrit d'Oxford ne me semble pas moins l'indice que le texte est soumis à une économie rhétorique serrée, où l'abrégé raisonné du texte biblique permet $\mathrm{d}^{\prime}$ '«aménager » la place prépondérante que tient l' auctoritas.

Quels niveaux d'auctoritas, et quelle Bible?

8 Au point où en sont les recherches, il est possible de distinguer deux ou trois niveaux d' auctoritas: l'ambiguïté tient au statut de l'éventuelle troisième catégorie identifiée, problème délicat qui mérite d'amples et prudentes vérifications. En premier lieu, il y a les éclaircissements introduits par la formule "c'est-à-dire » (" that is » ou " that is to sey »), qui portent essentiellement sur des points de lexique. Ils pourraient être de la seule autorité du compilateur. En second lieu, les citations extrêmement abondantes tirées (essentiellement) de Nicolas de Lyre et de la Glose dite "ordinaire ", les deux principales auctoritates en matière d'exégèse et de théologie $a u \mathrm{XIV}^{\mathrm{e}}$ siècle $^{7}$, sont identifiées par les noms propres mêmes. Elles tissent un lien serré entre le texte et ses sources d'autorisation, induisant un rapport scholastique à la Bible, dans un contexte d'efflorescence de la production religieuse en langue anglaise. En dernier lieu, de nombreux autres segments de texte, assez substantiels en général, ne sont pas même identifiés par les noms des auctoritates: quelques sondages comparatifs dans le texte le plus courant de la Glose (telle qu'elle se trouvait reproduite dans les marges de nombreux manuscrits de la Bible latine), me conduisent à penser pour le moment que la dette envers ce texte, et envers ceux de Nicolas de Lyre, est plus grande qu'il n'y parait. Devant le caractère encore hypothétique de ces résultats, il convient, dans le dernier mouvement de ce propos, de se concentrer non pas tant sur les niveaux de commentaire que sur les fragments bibliques qui les entourent. 
9 Le format et la nature du compendium laissent à penser que, selon toute vraisemblance, il n'a pas été conçu pour «tenir debout » tout seul. Ce n'est pas que sa lecture n'ait aucun sens, s'il est pris tel qu'en lui-même pour ce qu'il offre; mais les fins herméneutiques auxquelles revient l'usage si appuyé de Nicolas de Lyre et de la Glose méritent à l'évidence plus que l'exploration embryonnaire qui en existe à présent. Quoi qu'il en soit, il est loisible d'imaginer que, pour un utilisateur médiéval comme pour un lecteur moderne, la matière biblique ici exposée ait pris son véritable relief en se retrouvant confrontée à un texte complet de la Bible. Ajoutons que la petite taille du manuscrit (les folios mesurent 208x140 mm) et de nombreuses indications de références à la Bible sur le modèle « Livre/ verset ", suggèrent que le compendium ait pu fonctionner comme un outil de travail et d'indexation du texte complet, susceptible de s'adjoindre à toute lecture vernaculaire. Une question demeure cependant : ce texte complet est-il une Bible Vulgate (en latin), ou une Bible déjà traduite en langue anglaise? La controverse autour de la légitimité d'une traduction en anglais, évoquée brièvement plus haut, a pris naissance avec les entreprises de traduction intégrale, issues de l'impulsion initiale donnée à Oxford par John Wyclif et le tout premier cercle des contestataires, à Queen's College, à la fin des années 1360 et au début de la décennie suivante. Les chercheurs s'accordent à distinguer deux versions, appelées simplement «première» («early») et "tardive» («late») qui se sont chevauchées sur une vingtaine d'années de manière extrêmement complexe ${ }^{8}$.

Pour évoquer cette question, tournons-nous vers les Psaumes, qui sont les textes parmi les plus densément commentés dans le compendium. Voici une grande partie du psaume XIII, ou plutôt son anatomie :

Dixit insipiens. The vnwise man seyd. Lira expounes this psalme of Nabugodonosor that blasfemed God of Israel in worde and dede when he comanded the temple to be brend by Nabuzardan, which thing he wold not hafe done if he hade holden hym verrey God; and so is this psalme partied in to iiij. First he describes the malice of Nabugod and of his peple. After ther The Lord fro heuen is how God examynd ther malice which knowes thoghtes and dedes of men. After ther Whether they shal not knowe is how the forseyd synners shal knowe the rightwisnes of God by experience of peyne which reproued the peple of Israel [...]. After ther Who shal gif is put the delyuering of Israel thorw the mercy of God . After the glose the intencion of the prophete here is to confounde the hardnes and the vnwisdam of the Iewes that belefe not to Criste now he is cumen whose comyng they desired. And it hace iij parties. First he blames the herdnes of the Iewes and telles ther malice. After ther Whether they shal not knowe he manasses what dome shal cum to them that do such wikkednes. [... $]^{9}$

11 Ce ne sont pas les éléments de polémique anti-juive qui retiendront notre attention, mais bien l'architecture du commentaire. Elle donne une image assez exemplaire de la manière par laquelle l'auteur procède dans chacun des psaumes. Ils commencent tous par les premiers mots du psaume en latin (ici soulignés). Suit le commentaire lui-même, manifesté par des marqueurs d'analyse qui guident la lecture du psaume dans son intégralité. Ces points d'articulation sont doubles: la citation des fragments bibliques proprement dits (figurés en gras), et l'appareil d'interprétation (mis en italique), issu de la lecture attentive de Nicolas de Lyre et de la Glose et qui compose un squelette discursif à l'ensemble. On notera la nature encore très spatiale des déictiques : "after ther ", "à partir de cet endroit ", le texte sacré est un lieu d'où l'on part et où l'on revient à loisir. D'ailleurs, le commentaire fait retour sur les mêmes points d'articulation dans la lecture : le passage commençant par "Whether they shal not knowe » est ainsi exposé une première fois en suivant Lyre, puis revisité à l'aide de la Glose. Dans ce maillage serré, on s'attachera seulement à comparer les fragments bibliques en gras avec leurs correspondants, dans un 
représentant relativement « fiable » de chacune des deux versions ${ }^{10}$. Le manuscrit Bodley 959, représentant de la "première version ", donne les syntagmes suivants : "The vnwise man seide [...] The lord fro heuene [...] Whether alle shul not knowen [...] Who shal gyuen [...] »" Un représentant de la version dite "tardive », le manuscrit Oxford, Bodleian Library, Bodley 554, offer : "The unwise man seide/The lord biheld fro heuene/Wher alle men that worchen wickidnesse shulen not knowe/who shal gyue $»^{12}$. Il ressort de cette comparaison une communauté de formulations, mais pas de correspondances absolues, ce qui me conduit à postuler que les segments bibliques représentent peut-être une traduction indépendante du compilateur du compendium.

En guise de conclusion : l'écriture de la traduction

La finalité exacte de cet unique manuscrit n'est pas élucidée. Ses liens avec la mouvance lollarde semblent probants, pour peu qu'on pose clairement les conditions du rapprochement. En l'occurrence, les lemmes bibliques sont peut-être des traductions originales, au sein d'un environnement textuel où domine l'exégèse latine mise en anglais. S'il est vrai que l'on s'attache toujours à distinguer ce qui fait la spécificité d'une pratique, et que les traits originaux dégagés fondent une écriture, j'appelle ici écriture l'activité herméneutique du compilateur anonyme. Les conditions de cette activité d'abréviation et de commentaire constituent l'écriture d'une traduction. Elle laisse entendre combien le « traduire » en vernaculaire, et singulièrement ici, le « traduire » biblique, est à la croisée d'au moins deux chemins : celui de la rhétorique qui a gagné en puissance discursive, et celui des auctoritates tutélaires exposées in sermone anglico.

\section{BIBLIOGRAPHIE}

COPELAND Rita, Rhetorics, Hermeneutics, and Translation in the Middle Ages. Academic Traditions and Vernacular Texts, Cambridge, Cambridge University Press, Cambridge Studies in Medieval Literature 11, 1991.

DAHAN Gilbert, L'Exégèse chrétienne de la Bible en Occident médiéval XII ${ }^{e}-X I V^{e}$ siècle, Paris, Cerf, « Patrimoines Christianisme », 1999.

LAMBERT Malcolm, Medieval Heresy. Popular Movements from the Gregoriam Reform to the Reformation, seconde édition, Oxford, Blackwell, 1992.

LINDBERG Conrad, éd., MS Bodley 959. Genesis-Baruch 3.20 in the Earlier Version of the Wycliffite Bible. Vol.4 : Esdras-Ecclesiasticus 48.6, Stockholm, Almqvist \& Wiksell, « Stockholm Studies in English $13 », 1965$.

MinNiS, A. J., Medieval Theories of Authorship. Scholastic Literary Attitudes in the Later Middle Ages, seconde édition, Aldershot, Wildwood House, 1988.

Oxford, Bodleian Library, MS Bodley 554.

REILLY Robert, A Middle English Summary of the Bible: An Edition of Trinity College (Oxon) MS 93, thèse inédite, Université de Washington, 1966. 
WATSON Nicholas, « Censorship and Cultural Change in Late-Medieval England : Vernacular Theology, the Oxford Translation Debate, and Arundel's Constitutions of 1409 ", Speculum, $\mathrm{n}^{\circ} 70$, 1995, 822-864.

\section{NOTES}

*. Cet article est la traduction en français (avec de substantielles modifications) de la communication prononcée en anglais par l'auteur à la sixième Conférence internationale sur la théorie et la pratique de la traduction au Moyen Âge (Sixth Cardiff Conference on the Theory and Practice of Translation in the Middle Ages), Saint-Jacques de Compostelle, juillet 2001. Voir dans ce même numéro le compte rendu de cette conférence. Elle a bénéficié de la discussion qui a suivi la fin de l'atelier où la communication a été présentée ; l'auteur tient à remercier les intervenants de cette discussion, notamment A. J. Minnis (University of York) et Diane Speed (University of Sidney).

1. L'expression « mise en anglais » correspond à la perspective (et au titre) d'un travail récent de David Lawton, «Englishing the Bible, 1066-1519 ", dans The Cambridge History of Medieval English Literature, éd. David Wallace, Cambridge, Cambridge University Press, 1999, p. 454-482.

2. N. R. Ker, «A Middle English Summary of the Bible », Medium Ævum, n 29, 1960, 115-118; Id., Medieval Manuscripts in British Libraries, vol. III, Oxford : Clarendon, 1983, p. 713. Voir aussi S. J. Ogilvie Thomson, The Index of Middle English Prose. Handlist VIII. Manuscripts Containing Middle English Prose in Oxford College Libraries, Cambridge, D. S. Brewer, 1991, p. 101.

3. Sur le contexte général, voir Nicholas Watson, « Censorship and Cultural Change in Late-Medieval England : Vernacular Theology, the Oxford Translation Debate, and Arundel's Constitutions of 1409 ", Speculum, n 70, 1995, 822-864. Pour une vue d'ensemble sur John Wyclif et les Lollards, on pourra se reporter notamment à Malcolm Lambert, Medieval Heresy. Popular Movements from the Gregorian Reform to the Reformation, seconde édition, Oxford, Blackwell, 1992, p. 225-283.

4. Biblia Sacra Vulgata, éds. R. Weber et R. Gryson, Stuttgart, Deutsche Bibelgesellschaft, $4^{\mathrm{e}}$ édition, 1994, p. 1001.

5. MS Trinity 93, fol. 74v (Reilly, Middle English Summary, p. 182). Je n'adopte pas la même ponctuation que Reilly. Pour des raisons de commodité, je translitère la rune appelée «thorn » en «th».

6. Rita Copeland, Rhetoric, Hermeneutics, and Translation in the Middle Ages. Academic Traditions and Vernacular Texts, Cambridge : Cambridge University Press, 1991, p. 23-24 (sur les débuts de l'ambiguïté autour des éléments constitutifs de la rhétorique chez Quintilien, Institution oratoire, I, 9, 2-6) et p. 197-201 (sur l'usage de l'abbreviatio par Chaucer dans sa Légende des femmes vertueuses).

7. Sur la Glose, voir Beryl Smalley, The Study of the Bible in the Middle Ages, Oxford, Blackwell, $3^{\mathrm{e}}$ édition, 1983, p. 46-66, et Gilbert Dahan, L'Exégèse chrétienne de la Bible en Occident médiéval XII -XIV siècle, Paris, Cerf, « Patrimoines Christianisme », 1999, passim. Le franciscain Nicolas de Lyre (ca 1270-1340) est l'auteur de deux commentaires sur toute la Bible, appelés « Postilles » (Postillae super totam Bibliam), dont le succès a été immense du vivant de leur auteur. Sur sa vie et l'importance de son œuvre, outre les deux références précitées, voir notamment A. J. Minnis, Medieval Theory of Authorship. Scholastic Literary Attitudes in the Later Middle Ages, Aldershot, Wildwood House, 1988 ; « Scriptural Science 
and Signification : From Alexander's Sum of Theology to Nicholas of Lyre ", dans Medieval Literary Theory and Criticism c. 1000-c. 1375. The Commentary Tradition, éds. A. J. Minnis, A. B. Scott et D. Wallace, Oxford, Clarendon, 1988, p. 197-276 (notamment p. 266-276) ; Nicholas of Lyra: The Senses of Scripture, éds. Philip D. W. Krey et Lesley Smith, Leyde, Boston, Cologne, Brill, 2000.

8. Voir plus loin pour quelques références bibliographiques relatives aux deux versions de la Wyclif Bible.

9. MS Trinity 93, fol. 38r ; Reilly, Middle English Summary, p. 95. Je suis Reilly dans l'ajout de [mey] : Dixit insipiens. L'homme insensé a dit. Lire expose ce psaume [comme parlant] de Nabucodonosor qui a blasphémé le dieu d'Israël en paroles et en actes lorsqu'il a commandé que le temple soit brûlé par Nabuzardan, chose qu'il n'aurait pas faite s'il l'avait tenu pour le vrai Dieu; ce psaume se divise ainsi en quatre parties. En premier lieu, il décrit la méchanceté de Nabuco[donosor] et de son peuple. À partir de cet endroit Le Seigneur depuis le ciel se trouve comment Dieu a sondé leur méchanceté, qui connait les pensées et les actes des hommes. À partir de cet endroit S'ils ne connaîtront pas se trouve comment lesdits pécheurs connaîtront la justice de Dieu par l'expérience du tourment qui a réprouvé le peuple d'Israël [...]. À partir de cet endroit Qui donnera est présentée la délivrance d'Israël à travers la miséricorde de Dieu. D'après la Glose l'intention du prophète ici est de confondre la dureté et la folie des Juifs qui ne croient pas en le Christ après sa venue, bien qu'ils eussent désiré cette venue. Et il comporte trois parties. En premier lieu il blâme la dureté des Juifs et exprime leur méchanceté. À partir de cet endroit S'ils ne connaîtront pas il agite la menace du jugement divin qui s'abattra sur ceux qui font tant de mal. [...]

10. Le critère de « fiabilité » des versions est un problème trop délicat pour qu'il soit possible de l'aborder ici, et il n'existe pas de référence bibliographique unique à laquelle se référer sur ce point. Je me contenterai de renvoyer à Sven L. Fristedt, The Wycliffe Bible, 2 vol., Stockholm, Almqvist \& Wiksell, 1953-1963 ; aux remarques passim dans les introductions à chacun des volumes de Conrad Lindberg (voir note suivante pour l'un d'entre eux) ; et à Michael Wilkes, « Misleading Manuscripts : Wyclif and the NonWycliffite Bible ", Studies in Church History, n 11, 1975, 147-61.

11. Conrad Lindberg, éd., MS Bodley 959. Genesis - Baruch 3.20 in the Earlier Version of the Wycliffite Bible.volume 4 : Esdras-Ecclesiasticus 48.6, Stockholm, Almqvist \& Wiksell, 1965, p. 173.

12. Oxford, Bodleian Library, MS Bodley 554, fol. $5 \mathrm{v}$. Voir la description sommaire dans F. Madan et H. H. E. Craster, Summary Catalogue of Western Manuscripts in the Bodleian Library at Oxford, Vol. II Part I, Oxford, Clarendon, 1922, p. 311. Voir les quelques remarques, sur les psaumes et leurs gloses, de Michael P. Kuczynski, Prophetic Song. The Psalms as Moral Discourse in Late Medieval England, Philadelphia, University of Pennsylvania Press, 1995, p. 71-6, et Id., « Rolle among the Reformers : Orthodoxy and Heterodoxy in Wycliffite Copies of Richard Rolle's Psalter » dans Mysticism and Spirituality in Medieval England, éds. William F. Pollard et Robert Boenig, Cambridge, D. S. Brewer, 1997, p. 191-195. 


\section{RÉSUMÉS}

Le manuscrit Oxford, Trinity College 93 contient le seul exemplaire connu d'une longue composition en prose moyen anglaise, qui intéresse au premier chef l'histoire de la théologie vernaculaire dans l'Angleterre de la fin $d u x v^{e}$ et du début $d u x^{e}$ siècle. Ce texte est à la fois un résumé et un commentaire de la Bible, deux polarités que l'auteur, sous les termes d'abbreviatio et auctoritas, érige en principes complémentaires d'écriture, l'écriture d'une traduction. Il propose l'analyse rapide des principaux éléments qui ressortissent à ce double fonctionnement, tentant de la sorte d'entrevoir la place et l'originalité de ce texte, dans un contexte de répression de l'anglais comme langue propre à traduire-réécrire la Bible*.

Translating

Between Abbreviatio and Auctoritas : A Middle English Compendium to the Bible

MS Oxford, Trinity College 93 contains the unique known copy of a substantial Middle English prose composition which is of chief inter-est for the history of vernacular theology in latefourteenth and early-fifteenth century England. This text is both a summary of and commentary on the Bible, two discursive modes which the author chooses to label abbreviatio and auctoritas, and in which he sees two combining sources of "authorial" writing. He proposes an overview of the main constituents of this twofold system (including a quick comparison with Wycliffite versions of the biblical text), thereby attempting to map out the place and originality of the compendium, in a context of ecclesiastical repression exerted towards "englishing" the Bible.

\section{AUTEUR}

\section{JEAN-PASCAL POUZET}

Ancien élève de l'École Normale Supérieure, agrégé d'anglais, Jean-Pascal Pouzet est allocataire-moniteur à l'Université Paris IV-Sorbonne. Il prépare une thèse sur les traductions versifiées de la Bible dans la littérature moyen anglaise (XIII $-\mathrm{XV}^{\mathrm{e}} \mathrm{s}$.). En parallèle, le travail présenté ici est l'écho d'une recherche qu'il vient de commencer, portant sur les modes d'expression de la théologie vernaculaire dans les textes en prose. 